\title{
Widely metastatic glioblastoma with $B R C A 1$ and ARID1A mutations: a case report
}

Melissa Umphlett ${ }^{1}$, Stephanie Shea', Jessica Tome-Garcia', Yizhou Zhang², Adilia Hormigo ${ }^{3}$, Mary Fowkes', Nadejda M. Tsankova ${ }^{1,4}$ and Raymund L. Yong ${ }^{2^{*}}$ (D)

\begin{abstract}
Background: Glioblastoma (GBM) is a highly malignant brain neoplasm with poor survival. Despite its aggressive nature, metastatic spread of GBM is identified only rarely. While the molecular alterations associated with GBM and its subtypes are well-described, there remains a gap in understanding which alterations may predispose towards metastasis. In this report, we present a case of GBM with multi-organ metastases and discuss its genomic alterations.
\end{abstract}

Case presentation: A 74-year-old woman was diagnosed with left occipital glioblastoma (IDH-wildtype, MGMTunmethylated), for which she underwent resection, standard chemoradiation, and then stereotactic radiosurgery (SRS) for local recurrence. One month after SRS, work-up for a pathologic hip fracture revealed a left breast mass, lytic lesions involving pelvic bones, and multiple pulmonary and hepatic lesions. Biopsies of the breast and bone lesions both demonstrated metastatic IDH-wildtype GBM. For worsening neurologic symptoms, the patient underwent debulking of a large right temporal lobe recurrence and expired shortly thereafter. Autopsy confirmed metastatic GBM in multiple systemic sites, including bilateral lungs, heart, liver, thyroid, left breast, small bowel, omentum, peritoneal surfaces, visceral surfaces, left pelvic bone, and hilar lymph nodes. Targeted sequencing was performed on tissue samples obtained pre- and postmortem, as well as on cell cultures and an orthotopic mouse xenograft derived from premortem surgical specimens. A BRCA1 mutation (p.1571T) was the only variant found in common among the primary, recurrence, and metastatic specimens, suggesting its likely status as an early driver mutation. Multiple subclonal ARID1A mutations, which promote genomic instability through impairment of DNA mismatch repair, were identified only in the recurrence. Mutational spectrum analysis demonstrated a high percentage of C:G to T:A transitions in the post-treatment samples but not in the primary tumor.

Conclusion: This case report examines a rare case of widely metastatic IDH-wildtype GBM with a clonal somatic mutation in BRCA1. Post-treatment recurrent tumor in the brain and in multiple systemic organs exhibited evidence of acquired DNA mismatch repair deficiency, which may be explained by functional loss of ARIDIA. We identify a potential role for immune checkpoint and PARP inhibitors in the treatment of metastatic GBM.

Keywords: Glioblastoma, Metastasis, BRCA1 mutation, ARID1A mutation, Mismatch repair deficiency, Temozolomide, Treatment resistance

\footnotetext{
* Correspondence: raymund.yong@mountsinai.org

${ }^{2}$ Departments of Neurosurgery and Oncological Sciences, Icahn School of

Medicine at Mount Sinai, New York, NY, USA

Full list of author information is available at the end of the article
}

(c) The Author(s). 2020 Open Access This article is distributed under the terms of the Creative Commons Attribution 4.0 International License (http://creativecommons.org/licenses/by/4.0/), which permits unrestricted use, distribution, and reproduction in any medium, provided you give appropriate credit to the original author(s) and the source, provide a link to the Creative Commons license, and indicate if changes were made. The Creative Commons Public Domain Dedication waiver (http://creativecommons.org/publicdomain/zero/1.0/) applies to the data made available in this article, unless otherwise stated. 


\section{Background}

Glioblastoma (GBM) is the most common primary brain tumor in adults and universally harbors a poor prognosis due to its aggressive nature [1]. Despite modern improvements in treatment for afflicted patients, the mortality of GBM remains high, with a median overall survival of 10-16.5 months [2]. Although it is commonly associated with widespread infiltration throughout the brain, GBM is only rarely associated with extracranial metastatic disease $[3,4]$, which occurs at an estimated incidence of less than 2\% [5-11]. Widespread multiorgan metastases are rarer still. A literature review of 79 cases of extracranial metastatic GBM found that only $4 \%$ of cases examined had greater than four metastatic sites [12]. Furthermore, to our knowledge there are only nine reported cases of high-grade glioma metastases involving skin, soft tissue, or muscle [13].

Possible explanations for the rarity of GBM systemic metastases include underdiagnosis and short patient survival time [14]. Case reports have described the diagnosis of metastatic GBM in recipients of lung, liver, and other organ transplants from deceased donors harboring GBM, indicating that GBM micrometastases may be present at the time of death $[14,15]$. Such cases suggest that rates of clinically detected GBM metastases may underestimate the extent to which these malignant tumors are capable of seeding distant organs. The underlying genomic drivers of systemic GBM metastases remain poorly defined. Limited molecular analyses of several reported cases have suggested an association with mutations in TP53 [16]; however, TP53 mutations are also among the most common across all cancer.

Recognizing the importance of identifying unique molecular features that may drive extracranial GBM metastasis, we present a rare case with widespread multi-organ metastases, placing special attention on a comparative analysis of the most frequent genetic alterations found in the primary tumor, its post-treatment brain recurrence, and multiple systemic metastatic sites.

\section{Case presentation}

A 74-year-old female was initially evaluated for a headache and right eye peripheral vision loss. MRI brain with and without contrast was performed, revealing a $5.5 \mathrm{~cm}$ heterogeneously T2 hyperintense lesion with thick irregular nodular enhancement in the left parietaloccipital region (Fig. 1). The patient underwent a gross total resection of the mass that was diagnosed as GBM, IDH-wildtype, WHO grade IV, MGMT promoter methylation not detected. Fresh specimen in multiple sectors was processed for tumor culture. Following resection, she received hypofractionated concurrent chemoradiation with temozolomide followed by four cycles of adjuvant temozolomide (TMZ). Six months later, the patient developed multifocal GBM recurrence in the right temporal and frontal lobes, for which she underwent single fraction 18 Gy stereotactic radiosurgery to the right frontal lesion and five fractions of 2250 cGy to the right temporal lesion.

Three months following SRS, the patient began to experience falls associated with hip pain and difficulty walking. MRI of the right hip demonstrated a pathologic hip fracture, which was thought to be due to metastatic disease from an undiagnosed second primary cancer. The patient then developed altered mental status and right-sided upper motor neuron facial weakness. A full metastatic imaging work-up was performed, revealing a $3.9 \mathrm{~cm}$ left breast mass, multiple lytic lesions of the pelvic bones, and multiple pulmonary and hepatic nodules. Core biopsies were obtained from the left breast and the left pubic bone, both of which demonstrated metastatic GBM.
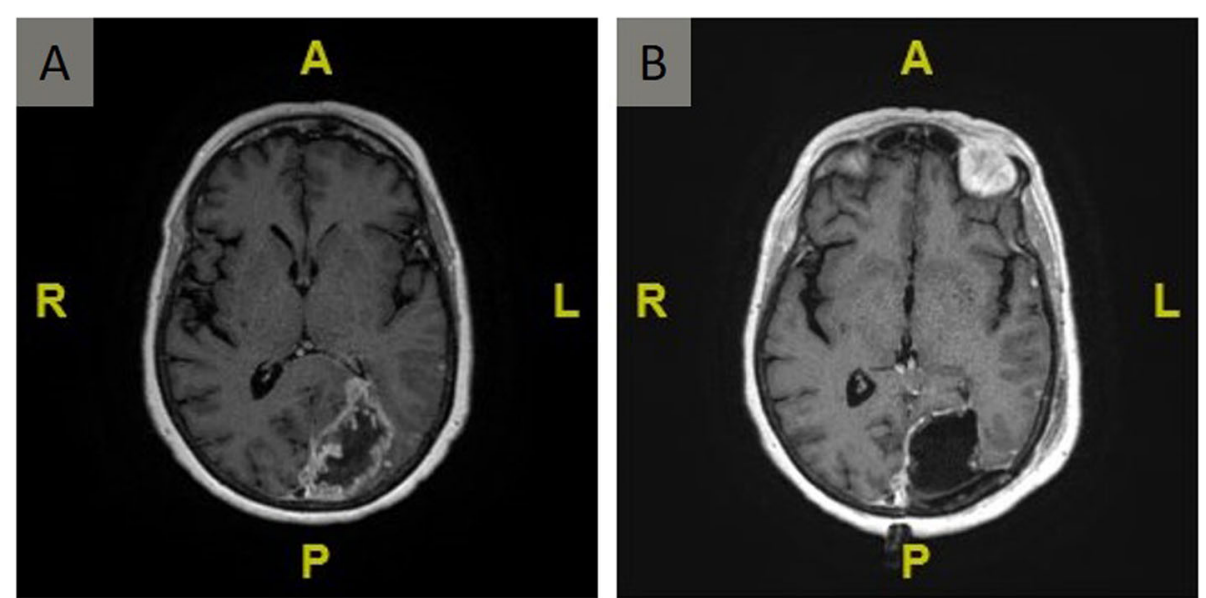

Fig. 1 Primary GBM in the left occipital lobe. Axial T1 gadolinium-enhanced magnetic resonance image performed (a) preoperatively, and (b) 24 h postoperatively 
The patient's mental status deteriorated as the right temporal recurrence rapidly progressed, and she underwent right temporal craniotomy for debulking of the tumor eleven months after her initial diagnosis of GBM (Fig. 2). Histologically, this secondary tumor was identical to the primary. $2 \times 10^{5}$ freshly dissociated cells from the right temporal recurrence were orthotopically transplanted directly into the striatum of SCID mice with preserved microglial activity (IcrTac:ICR-Prkdc ${ }^{\text {scid }}$ strain) to assess the cells' ability to generate a patient-derived xenograft (PDX). Following surgery, the patient stabilized neurologically, but opted for palliative care and was transferred to hospice where she expired one month later.

While the patient was alive, consent was obtained for a rapid autopsy, which was ultimately performed within four hours of death. Gross and histological evaluation confirmed numerous GBM metastases. The extent of metastatic disease was widespread, including bilateral lungs, heart, liver, thyroid, left breast, small bowel, omentum, peritoneum, left pelvic bone, and hilar lymph nodes (Fig. 3). Notably, there were extensive metastatic lesions involving the abdominal cavity.

The autopsy revealed residual GBM in the original site of occurrence (left occipital lobe). On histological examination of pre- and post-mortem samples, the metastases appeared identical to the primary tumor and temporal recurrence. All sites demonstrated the classic appearance of GBM on H\&E staining, including nuclear atypia, microvascular proliferation, and pseudopalisading necrosis. Sarcomatous transformation was not identified on histological review of the tissue sections, and was confirmed absent by reticulin-staining performed on representative sections from occipital lobe, temporal lobe
(Fig. 2e), left breast, heart, lung, liver, lymph nodes, and omentum.

To evaluate the molecular phenotype of the primary, recurrent, and metastatic lesions, two different nextgeneration-sequencing (NGS) panels were employed (see Additional file 1). The Ion AmpliSeq Hotspot Cancer NGS Panel v2, covering 50 genes and 207 amplicons, was performed on all pre-mortem (left occipital brain primary, right temporal lobe recurrence, and left breast metastases) and select post-mortem (paratracheal lymph node and omentum) tissue specimens. The Ion Torrent Oncomine Comprehensive Assay v3, covering 161 cancer driver genes, was performed on the remaining postmortem samples (left occipital lobe, right temporal lobe, left breast, lungs, and liver) collected during rapid autopsy.

The AmpliSeq panel revealed single nucleotide variants (SNVs) in PIK3CA, SMARCB1, BRAF, and TP53. Notably, five different SNVs were detected for TP53. There were differences in TP53 mutations between the primary tumor, metastases, and among the metastases themselves (Fig. 4). The more comprehensive Oncomine panel revealed only one non-silent SNV common to all specimens: BRCA1 p.I571T. A large number of private mutations were detected in the temporal lobe recurrence, left breast, lung, and liver metastases. Among the extracranial metastatic sites, only the left breast, liver, and omentum specimens were found to share any mutations other than the one identified at BRCA1.

Cells cultured from the left occipital primary site at the time of initial diagnosis, and the right temporal site at the time of recurrence, expanded readily in serumfree conditions and were all found to harbor the $B R C A 1$ p.I571T mutation (see Additional file 1). Both mice, orthotopically xenotransplanted with cells from the
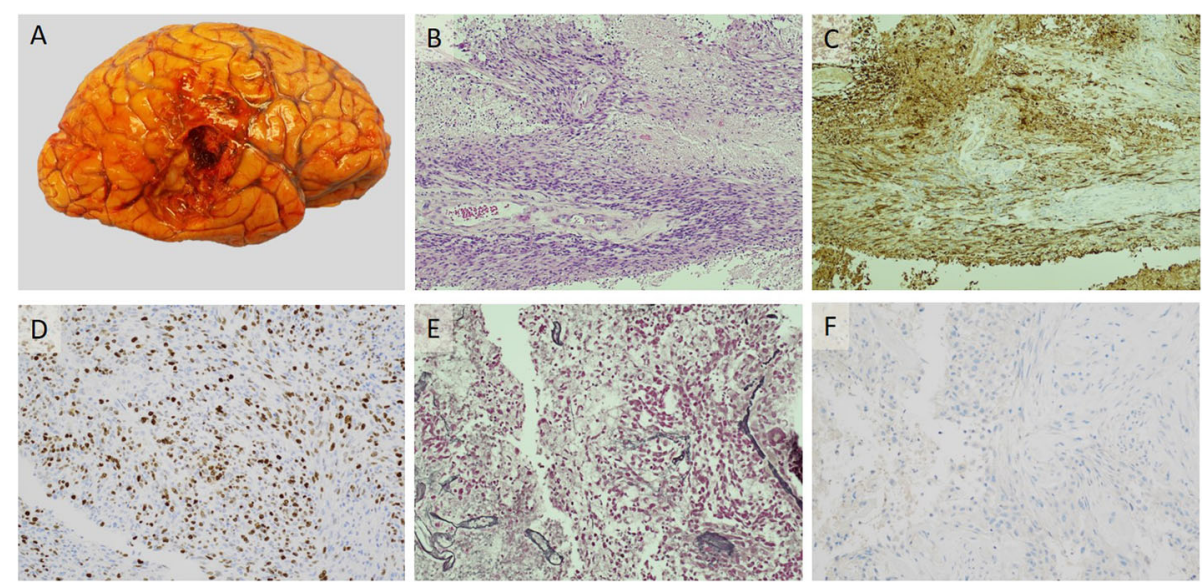
resection cavity $(6.0 \times 3.5 \mathrm{~cm})$ and residual tumor. $\mathbf{b}$ Tumor is histologically compatible with GBM on H\&E stain. c Tumor cells are diffusely positive on GFAP stain. $\mathbf{d}$ Tumor cells demonstrate positive MIB-1 focally up to $60 \%$. e Tumor cells are negative on reticulin stain. $\mathbf{f}$ Tumor cells are negative (wild-type) on IDH1 R132H stain. Micrographs are 10X magnification 

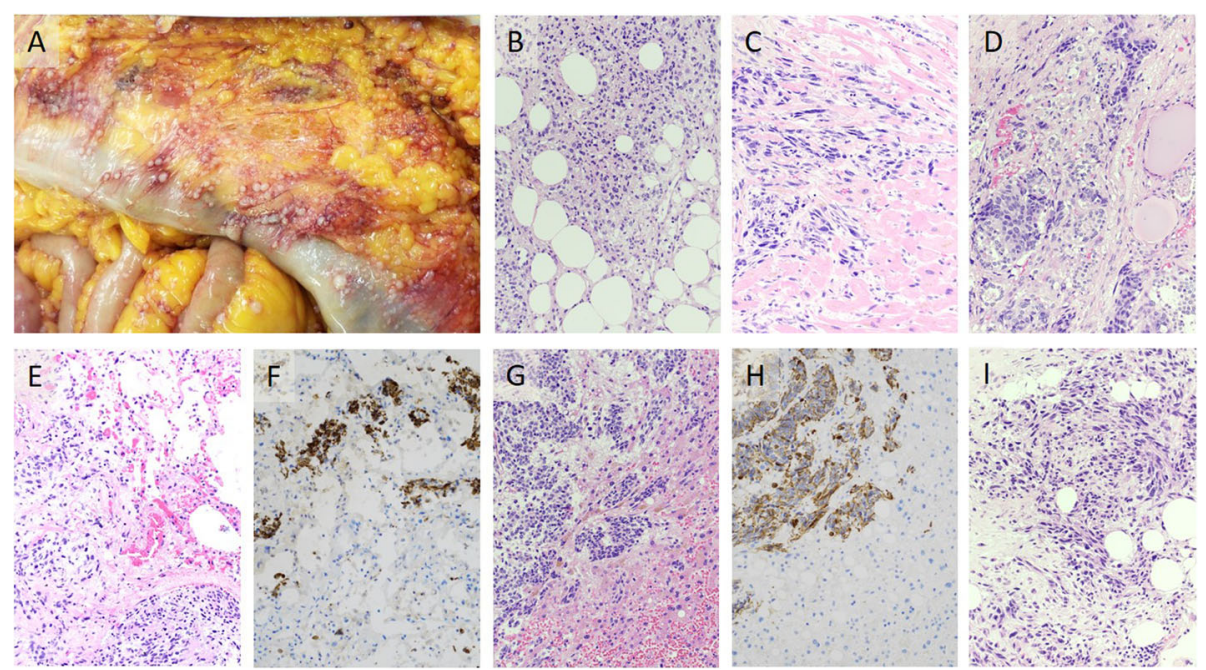

Fig. 3 Metastatic GBM lesions involving multiple organs. a Gross photo of "peritoneal glioblastomatosis," i.e., metastatic GBM studding the surface of the small bowel and omentum. b Metastatic GBM infiltrating breast tissue (H\&E stain). c Metastatic GBM infiltrating cardiac muscle (H\&E stain). d Metastatic GBM infiltrating thyroid parenchyma (H\&E stain). Metastatic GBM infiltrating lung tissue (e H\&E stain, and f GFAP stain). Metastatic GBM infiltrating breast tissue (g H\&E stain, and $\mathbf{h}$ GFAP stain). i Metastatic GBM infiltrating omental tissue (H\&E stain). Micrographs are 10X magnification

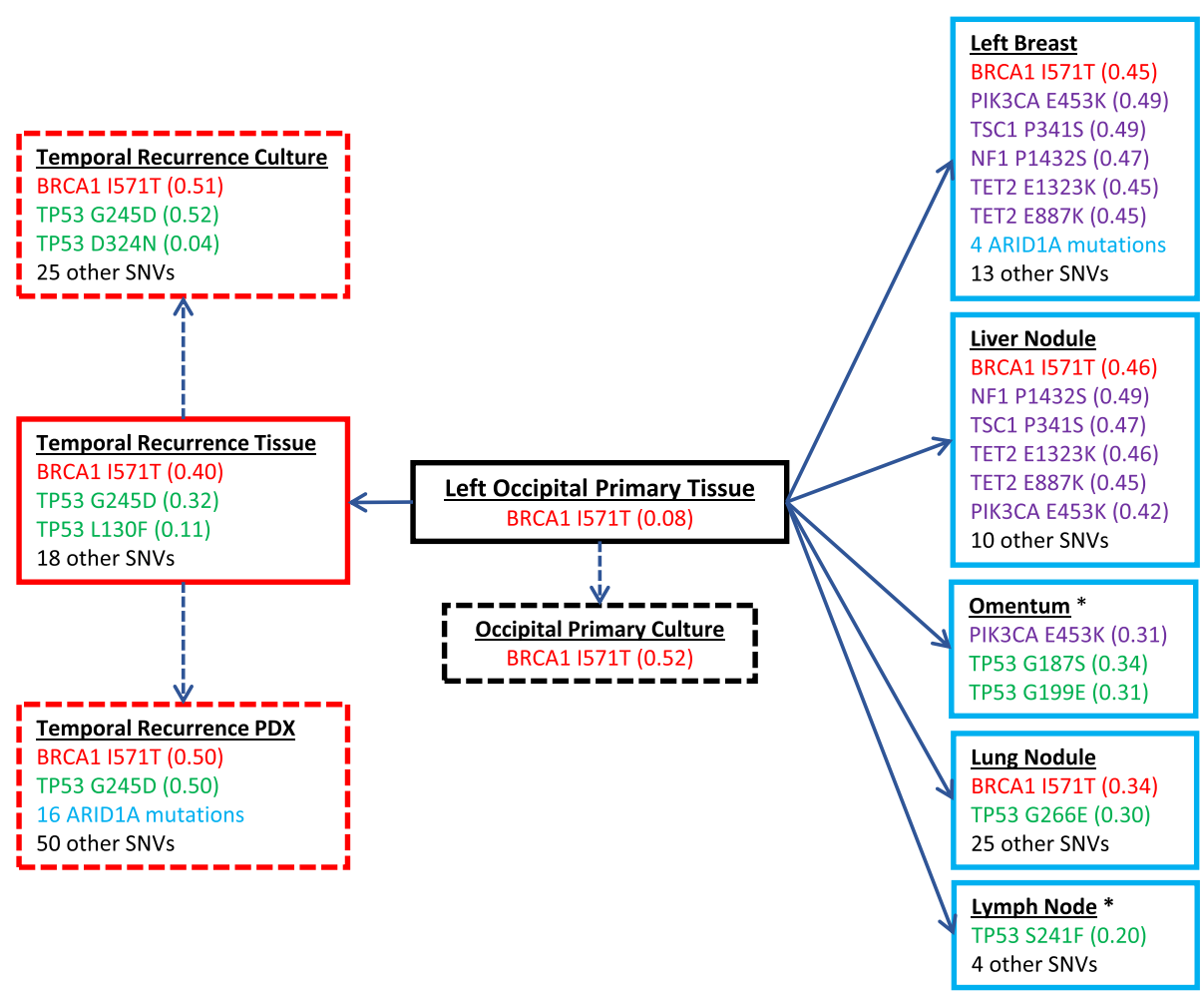

Fig. 4 Comparative analysis of coding SNVs identified in metastatic GBM tissue and derived cultures. BRCA1 p.I571T, an early putative driver mutation found at all time points -- primary resection (black boxes), brain recurrence (red boxes), extracranial metastatic sites (blue boxes) -- is highlighted in red text. Several distinct TP53 SNVs (green text) were identified in the temporal recurrence and multiple metastatic sites, suggesting convergent evolution. Several SNVs (purple text) were shared between metastatic sites, indicating a common ancestral clone. To assist in distinguishing putative driver and passenger mutations, variant frequencies (in brackets) were compared between source tissue (solid boxes) and cultured or xenografted cells (dashed boxes). Asterisks indicate samples sequenced using Ampliseq Cancer Hotspot panel only 
temporal recurrence, developed fatal malignant gliomas after 5 weeks, confirming the tumor's aggressive behavior in both human and rodents (Fig. 5). Necropsy analysis of the lungs, intestines, liver, and spleen in these mice did not reveal the presence of any peripheral metastases as seen in the patient. Tumor cells from this aggressive GBM were isolated from the primary PDX and were subsequently propagated in culture and used to generate a reliable PDX model which forms within 3-4 weeks post implantation and, importantly, recapitulates both the rapid growth and the malignant infiltrative spread of human GBM. Mutational hotspot analysis of the PDX confirmed the presence BRCA1 p.I571T in all cells, and multiple inactivating mutations of ARID1A in a significant subpopulation. ARID1A mutations were also identified in the left breast autopsy specimen (Fig. 4).

To investigate whether the relatively high number of SNVs in all secondary sites compared to the primary could have resulted from mismatch repair (MMR) deficiency, we performed a mutational spectrum analysis; results confirmed a high proportion of C:G to T:A transitions, which is typical of the mismatch repair deficiency described in the setting of temozolomide-treated recurrent GBM (Fig. 6). The integrity of the mismatch repair pathway was evaluated using immunohistochemistry. All metastatic specimens exhibited positive staining for anti-MLH1, anti$\mathrm{MSH}$, anti-MSH6, and anti-PMS2, indicating microsatellite stability (MSS). MSS was further confirmed with PCR of established microsatellite loci [17] (see Additional file 1). In addition, we evaluated DNA polymerase epsilon $(P O L E)$ using PCR, which showed no alterations at mutational hotspots within the coding region of the gene.

\section{Discussion and conclusions}

GBM is known to have aggressive biological behavior with poor survival outcomes [1], as demonstrated in

A

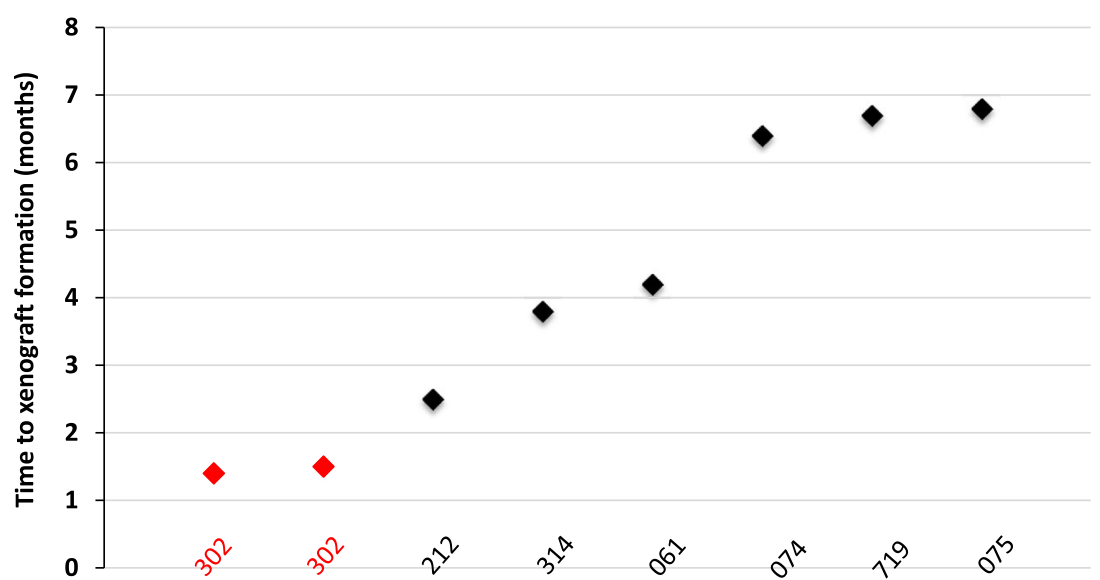

Xenograft Sample Numbers (De-Identified)

B

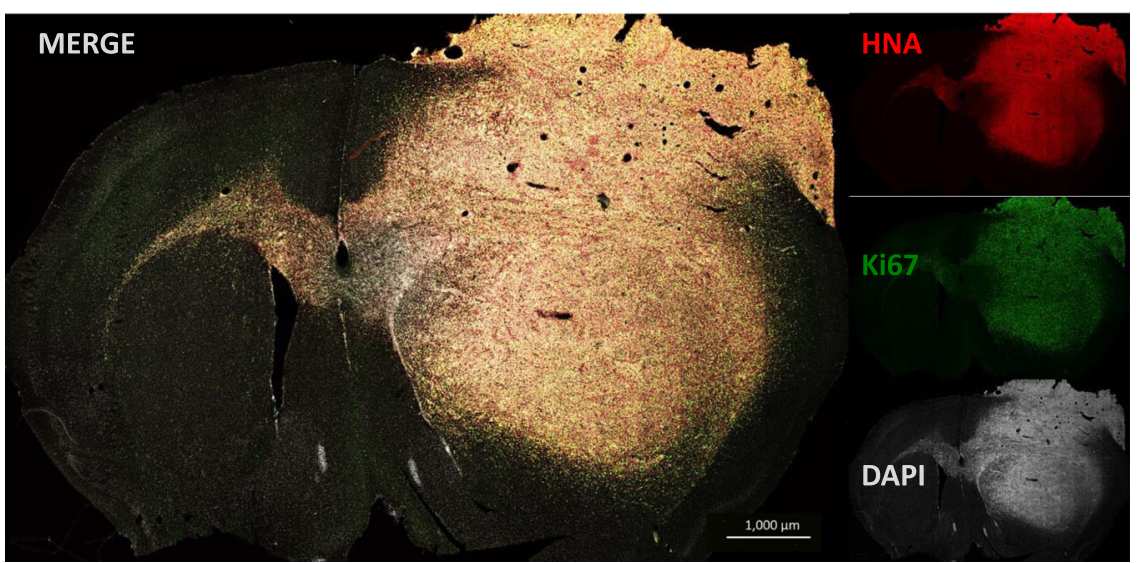

Fig. 5 Patient-derived xenograft of recurrent temporal lobe GBM in a SCID mouse brain. a Time to fatal xenograft formation for cells derived from the index case (sample ID 302) versus six other consecutive cases of IDH-wildtype GBM not associated with extracranial metastasis. b Micrograph of mouse brain 5 weeks after xenotransplantation into the right striatum of 200,000 GBM cells obtained during resection of the temporal lobe recurrence. Tumor cells are seen infiltrating the contralateral hemisphere via the corpus callosum. HNA, human nuclear antigen. Scale bar $=1000 \mu \mathrm{m}$. 


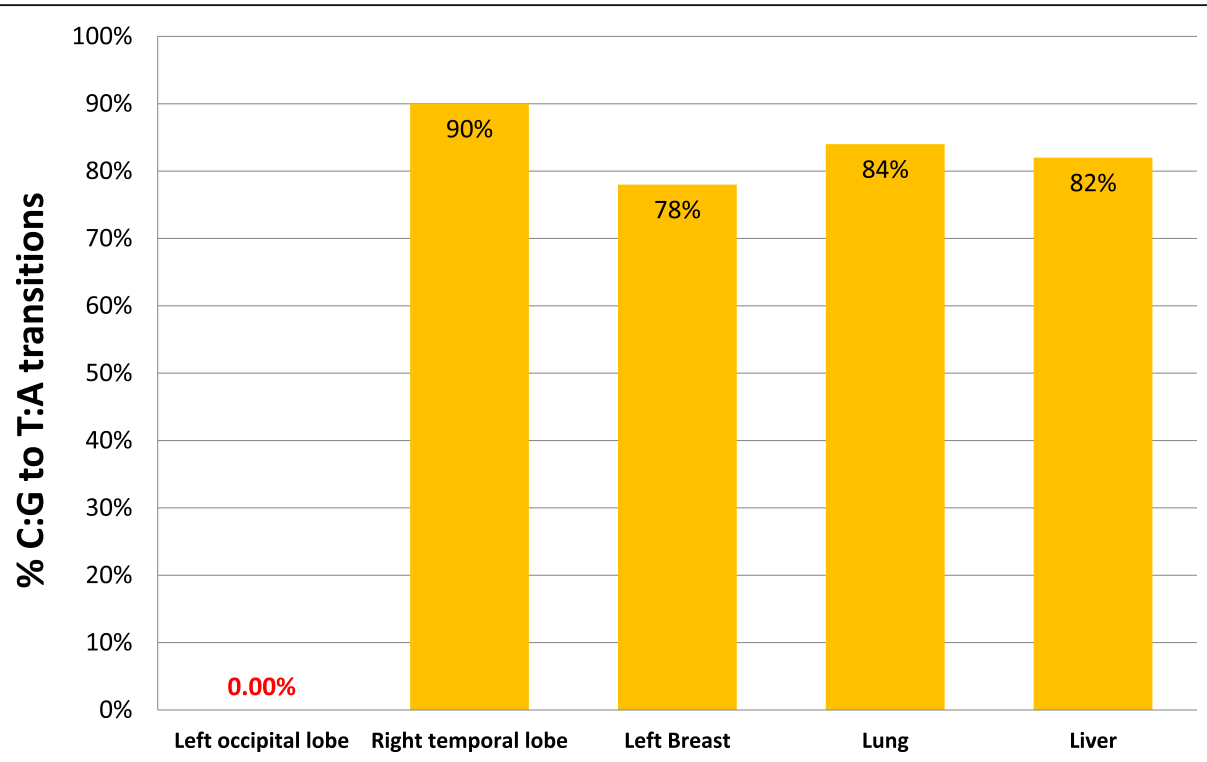

Anatomic Sites of Metastatic GBM Tissue Submitted for Mutational Hotspot Analysis

Fig. 6 Percentage of all somatic SNVs identified through targeted sequencing (Oncomine Comprehensive Assay version 2) of primary, recurrent, and metastatic GBM specimens that were C:G to T:A transitions

this case of a woman who expired approximately one year after initial diagnosis. At autopsy, in addition to the widespread extracranial metastases, this case had a unique gross finding. In the abdominal cavity, metastatic lesions extensively studded the surfaces of the small bowel, omentum, and peritoneum, mimicking the appearance of peritoneal carcinomatosis; therefore, when secondary to GBM, we propose the term "peritoneal glioblastomatosis" to describe this rare presentation (Fig. 3a).

While the molecular variants associated with GBM and its subtypes are well-described [18, 19], there remains a critical gap in understanding which genomic drivers may lead GBM to metastasize. The unusually large number of private SNVs observed in all specimens except the left-occipital primary suggests that a parental clone at the primary site acquired a hypermutator-like phenotype during adjuvant chemoradiation and subsequently seeded the extra-CNS sites, possibly via invasion of the sagittal sinus. The hypermutating subclone also seeded the contralateral temporal lobe - presumably via white matter migration - and was able to expand due to its location outside the primary radiation treatment volume. GBM dissemination via CSF pathways is also a recognized possibility, but in this case less likely to have been a major mechanism given the absence of diffuse leptomeningeal disease or spinal drop metastases.

Gliosarcoma is a rare variant of GBM with an increased tendency to metastasize [20], and must be considered in the differential diagnosis in this case. In gliosarcoma, metaplastic transformation of gliomatous tumor gives rise to a sarcomatous component, which is associated with a higher rate of connective tissue invasion and extracranial metastasis [20]. This transformation has been linked to the acquisition of driving TP53 mutations [21]. In our case, sarcomatous histology was not identified in any of the primary or secondary specimens; thus, the pathogenetic mechanism of extracranial spread of GBM in our case is likely distinct from that seen in gliosarcoma. Although numerous TP53 mutations were detected, none were shared between sites, suggesting they arose as a product of genetic instability in a parental clone, rather than as primary drivers of the instability. Consistent with this view, Park et al. detected multiple different TP53 mutations between sites in 2 out of a series of 6 metastatic GBM cases examined. They suggest that this resulted from dissemination of subclones that were dormant in the primary tumor, which then activated and expanded in the metastatic microenvironment [16].

Since it was the only coding alteration common to the primary tumor, recurrence, and metastases, we consider the BRCA1 p.I571T SNV to be the most likely driver of this GBM's unique metastatic phenotype. Although there is no literature to date describing a role for $B R C A 1 \mathrm{mu}$ tations in GBM pathogenesis, alterations in $B R C A 2$ have been associated with genomic instability in astrocytomas [22], and a BRCA2 inactivating mutation was found in the primary site of a metastatic GBM [23]. Perhaps screening for metastatic disease may be considered when 
BRCA mutations are found in a primary GBM. Among GBM specimens in The Cancer Genome Atlas (TCGA), $B R C A 1$ and BRCA2 missense mutations are rare, each occurring at a rate of $1.4 \%$. Piccirilli et al. [24] described a series of 11 patients with a history of invasive breast carcinoma who subsequently developed GBM; however, an analysis of $B R C A 1$ or $B R C A 2$ mutational status was not performed. BRCA1 defects are known to dysregulate cell checkpoint pathways and impair the fidelity of the DNA damage response, particularly to double-strand breaks (DSBs) [25]. We speculate that GBM cells with $B R C A 1$ defects might exhibit particularly high levels of genomic instability when exposed to DSB-inducing agents such as RT and temozolomide, increasing the risk of treatment-induced cancer evolution and acquiring new, aggressive phenotypes.

In GBM and other solid malignancies, a high proportion of acquired C:G to T:A transitions is classically associated with chronic exposure to alkylating agents in the context of a deficiency in one or more components of the DNA mismatch repair machinery. Possible mechanisms include acquired inactivating mutations or epigenetic silencing of the MMR genes MSH6, MSH2, MLH1, and PMS2. Recent studies show that treatment with TMZ of MGMT unmethylated tumors, such as in our case, introduces a strong selective pressure to lose mismatch repair pathway function [26]. Although immunostaining demonstrated intact MMR protein expression in the recurrent and metastatic specimens of our case, mutational hotspot analysis of the PDX derived from the temporal recurrence revealed inactivation of ARID1A, which has recently been shown to promote MMR by interacting with MSH2 [27]. ARID1A mutations are rare in GBM, occurring at a rate of $0.7 \%$ in newly diagnosed cases, and may be associated with an aggressive phenotype. Both cases described in TCGA were seen in males under the age of 50, one of whom survived less than 1 year. Thus, our case illustrates the need for caution in the treatment of MGMT unmethylated GBM with TMZ, even if the tumor exhibits microsatellite stability by conventional methods, since other forms of instability may exist.

In considering alternatives to TMZ, new data suggests that PARP inhibitor therapy may be effective in ARID1A- as well as BRCA-defective tumors [28]. ARID1A-defective tumors may also be particularly good candidates for immune checkpoint blockade due to the potentially large number of immune-activating neoepitopes generated by MMR deficiency [27]. The PARP inhibitors olaparib and BGB-290, among others, are currently being evaluated as radio- and chemosensitizers in both IDH-wildtype and IDH-mutant GBM in early phase clinical trials, but no molecular biomarkers for response have so far emerged [29-31].
In conclusion, we describe a rare and highly aggressive case of widely metastatic IDH-wildtype GBM with a clonal somatic mutation in BRCA1. Post-treatment recurrent tumor in the brain and in multiple systemic organs exhibited evidence of acquired DNA mismatch repair deficiency, despite retaining intact expression of mismatch repair pathway proteins. This may be explained by loss of ARID1A, which is required for MSH2 function.

\section{Supplementary information}

Supplementary information accompanies this paper at https://doi.org/10. 1186/s12885-020-6540-1.

Additional file 1. Supplementary Methods.

\section{Abbreviations}

GBM: Glioblastoma; MMR: Mismatch repair; MSS: Microsatellite stability; NGS: Next-generation sequencing; PDX: Patient-derived xenograft; SNV: Single nucleotide variant; TCGA: The Cancer Genome Atlas; TMZ: Temozolomide

\section{Acknowledgements}

We wish to thank Dr. Robert Sebra for generously providing resources for targeted sequencing; Dr. Michael Donovan, principal investigator of the Mount Sinai Cancer Institute Biorepository; Dr. Jane Houldsworth for interpretation of clinical sequencing data; and Dr. Amy Chan for her involvement in the care of the patient.

\section{Authors' contributions}

MU, SS, YZ, NMT and RLY were major contributors in writing the manuscript. YZ and RLY generated and interpreted genetic data. MU, SS, and NMT performed and interpreted all histology. JT-G and NMT generated and interpreted mouse xenograft data. MU and MF performed and interpreted the autopsy. AH, NMT, and RLY conceived the project. All authors read and approved the final manuscript.

\section{Funding}

This study was supported in part by R01NS106229 (N.M.T.) for collection and culture of tumor samples and R03NS104669 (N.M.T.) for generation of mouse xenografts.

\section{Availability of data and materials}

The data that support the findings of this study are not publicly available to protect patient privacy, but are available from the corresponding author upon reasonable request.

\section{Ethics approval and consent to participate}

The patient described in this case report consented to participate in the Mount Sinai Cancer Institute Biorepository IRB-approved protocol (study ID\# HSM\#-00135, Mount Sinai Health System IRB).

\section{Consent for publication}

Written informed consent for participation in the study and publication was obtained from the patient described in this case report.

\section{Competing interests}

The authors declare that they have no competing interests.

\section{Author details}

${ }^{1}$ Department of Pathology, Icahn School of Medicine at Mount Sinai, New York, NY, USA. ${ }^{2}$ Departments of Neurosurgery and Oncological Sciences, Icahn School of Medicine at Mount Sinai, New York, NY, USA. ${ }^{3}$ Department of Neurology, Medicine (Division Hem-Onc), Neurosurgery and the Tisch Cancer Institute, Icahn School of Medicine at Mount Sinai, New York, NY, USA. ${ }^{4}$ Department of Neuroscience, Icahn School of Medicine at Mount Sinai, New York, NY, USA. 
Received: 13 September 2019 Accepted: 13 January 2020

Published online: 20 January 2020

\section{References}

1. Saad AG, Sachs J, Turner CD, et al. Extracranial metastases of glioblastoma in a child: a case report and review of the literature. J Pediatr Hematol Oncol. 2007:72:157-61.

2. Rajagopala V, El Kamar FG, Thayparan R, Grossman ML. Bone marrow metastases from glioblastoma multiforme - a case report and review of literature. J Neuro-Oncol. 2005;72:157-61.

3. Schonsteiner SS, Bommer M, Haenle MM, et al. Rare phenomenon: liver metastases from glioblastoma multiforme. J Clin Oncol. 2011;29(23): e668-e71.

4. Lun M, Lok E, Gautam S, Wu E, Wong ET. The natural history of extracranial metastasis from glioblastoma multiforme. J Neuro-Oncol. 2011;105(2):261-73.

5. Waite K, Wharton SB, Old SE, Burnet NG. Systemic metastases of glioblastoma multiforme. Clin Oncol. 1999;11:205-7.

6. Datta CK, Weinstein JD, Bland JE, Brager PM, Stewart MA. A case of cervical lymph node metastasis resulting from glioblastoma multiforme. W V Med J. 1998;94:276-8

7. Fecteau $\mathrm{AH}$, Penn I, Hanto DW. Peritoneal metastasis of intracranial glioblastoma via a ventriculoperitoneal shunt preventing organ retrieval: case report and review of the literature. Clin Transpl. 1998:12:348-50.

8. Widjaja A, Mix H, Gölkel C, et al. Uncommon metastasis of a glioblastoma multiforme in liver and spleen. Digestion. 2000;61:219-22.

9. Yasuhara T, Tamiya T, Meguro T, et al. Glioblastoma with metastasis to spleen - case report. Neurol Med Chir. 2003:43:452-6.

10. Piccirilli M, Brunetto GM, Rocchi G, Giangaspero F, Salvati M. Extra central nervous system metastases from cerebral glioblastoma multiforme in elderly patients. Clinico-pathological remarks on our series of seven cases and critical review of the literature. Tumori. 2008;94:40-51.

11. Templeton A, Hofer S, Töpfer M, et al. Extraneural spread of glioblastoma report of two cases. Onkologie. 2008;31:192-4.

12. Kalokhe $\mathrm{G}$, et al. Metastatic glioblastoma: case presentations and a review of the literature. J Neuro-Oncol. 2012;107:21-7.

13. Armanios MY, Grossman SA, Yang SC, White B, Perry A, Burger PC, Orens JB. Transmission of glioblastoma multiforme following bilateral lung transplantation from an affected donor: case study and review of the literature. Neuro-Oncology. 2004;6:259-63.

14. Kraft M, Land F, Braunschweig R, Janzer RC. Parotid gland metastasis from glioblastoma multiforme: a case report and review of literature. Eur Arch Otorhinolarungol. 2008;265:709-11.

15. Chen $H$, Shah AS, Girgis RE, Grossman SA. Transmission of glioblastoma multiforme after bilateral lung transplantation. J Clin Oncol. 2008:26:3284-5.

16. Park CC, Hartmann C, Folkerth R, et al. Systemic metastasis in glioblastoma may represent the emergence of neoplastic subclones. J Neuropathol Exp Neurol. 2000;59:1044-50

17. Suraweera $\mathrm{N}$, et al. Evaluation of tumor microsatellite instability using five quasimonomorphic mononucleotide repeats and pentaplex PCR. Gastroenterol. 2002;123(6):1804-11.

18. Fratini $\vee$, et al. The integrated landscape of driver genomic alterations in glioblastoma. Nat Genet. 2013;45:1141-9.

19. Brenan CW, et al. The somatic genomic landscape of glioblastoma. Cell. 2013;155:462-77.

20. Ray A, et al. Extracranial metastasis of glioblastoma: three illustrative cases and current review of the molecular pathology and management strategies. J Molecul Clin Neurooncol. 2015;3:479-86.

21. Pain M, et al. Treatment-associated TP53 DNA-binding domain missense mutations in the pathogenesis of secondary gliosarcoma. Oncotarget. 2017; 9:2603-21.

22. Liu J, Lu H, Ohgaki H, Merlo A, Shen Z. Alterations of BCCIP, a BRCA interactive protein in astrocytomas. BMC Cancer. 2009;9:268.

23. Franceschi $\mathrm{S}$, et al. Molecular portrait of a rare case of metastatic glioblastoma: somatic and germline mutations using whole-exome sequencing. Neuro-Oncology. 2016;18(2):298-300. https://doi.org/10.1093/ neuonc/nov314.

24. Piccirilli M, Salvati M, Bistazzoni S, Frati A, Brogna C, Giangaspero F, Frati R, Santoro A. Glioblastoma multiforme and breast cancer: report on 11 cases and clinico-pathological remarks. Tumori. 2005;91:256-60.
25. Saha J, Davis AJ. Unsolved mystery: the role of BRCA1 in DNA end-joining. J Radiat Res. 2016:57(S1):24. https://doi.org/10.1093/jrr/rrw032.

26. Choi S, Yao Y, Grimmer M, Wahl M, Chang S, Costello. Temozolomide hypermutation in Gliomas. Neuro-Oncology. et al., 2018. https://doi.org/10. 1093/neuonc/noy016.

27. Shen J, et al. ARID1A deficiency promotes mutability and potentiates therapeutic antitumor immunity unleashed by immune checkpoint blockade. Nat Med. 2018;24:556-62.

28. Shen J, et al. ARID1A deficiency impairs the DNA damage checkpoint and sensitizes cells to PARP inhibitors. Cancer Discov. 2015 Jul;5(7):752-67. https://doi.org/10.1158/2159-8290.CD-14-0849.

29. Lesueur P, Lequesne J, Grellard JM, et al. Phase I/lla study of concomitant radiotherapy with olaparib and temozolomide in unresectable or partially resectable glioblastoma: OLA-TMZ-RTE-01 trial protocol. BMC Cancer. 2019 Mar;19(1):198. https://doi.org/10.1186/s12885-019-5413-y.

30. Sistigu A, Manic G, Obrist F, Vitale I. Trial watch - inhibiting PARP enzymes for anticancer therapy. Mol Cell Oncol. 2015;(2):e1053594. https://doi.org/10. 1080/23723556.2015.1053594

31. Pain $M$, Wang $H$, Lee $E$, et al. Treatment-associated TP53 DNA-binding domain missense mutations in the pathogenesis of secondary gliosarcoma. Oncotarget. 2018;9(2):2603-21.

\section{Publisher's Note}

Springer Nature remains neutral with regard to jurisdictional claims in published maps and institutional affiliations.
Ready to submit your research? Choose BMC and benefit from:

- fast, convenient online submission

- thorough peer review by experienced researchers in your field

- rapid publication on acceptance

- support for research data, including large and complex data types

- gold Open Access which fosters wider collaboration and increased citations

- maximum visibility for your research: over $100 \mathrm{M}$ website views per year

At BMC, research is always in progress.

Learn more biomedcentral.com/submissions 